\title{
Reduction of Mosquito Survival in Mice Vaccinated with Anopheles stephensi Glucose Transporter
}

\author{
J. Couto, ${ }^{1}$ S. Antunes, ${ }^{1}$ J. Ferrolho, ${ }^{1}$ J. de la Fuente, ${ }^{2,3}$ and A. Domingos ${ }^{1}$ \\ ${ }^{1}$ Global Health and Tropical Medicine, Instituto de Higiene e Medicina, Universidade Nova de Lisboa (GHMT-IHMT-UNL), \\ Rua da Junqueira 100, 1349-008 Lisboa, Portugal \\ ${ }^{2} \mathrm{SaBio}$, Instituto de Investigación en Recursos Cinegéticos (IREC), CSIC-UCLM-JCCM, Ronda de Toledo s/n, \\ 13005 Ciudad Real, Spain \\ ${ }^{3}$ Department of Veterinary Pathobiology, Center for Veterinary Health Sciences, Oklahoma State University, \\ Stillwater, OK 74078, USA
}

Correspondence should be addressed to S. Antunes; santunes@ihmt.unl.pt

Received 21 April 2017; Accepted 12 June 2017; Published 19 July 2017

Academic Editor: Yu-Chang Tyan

Copyright (C) 2017 J. Couto et al. This is an open access article distributed under the Creative Commons Attribution License, which permits unrestricted use, distribution, and reproduction in any medium, provided the original work is properly cited.

\begin{abstract}
Despite the fact that recent efforts to control/eradicate malaria have contributed to a significant decrease in the number of cases and deaths, the disease remains a global health challenge. Vaccines based on mosquito salivary gland antigens are a potential approach for reducing vector populations and malaria parasites. The Anopheles AGAP007752 gene encodes for a glucose transporter that is upregulated during Plasmodium infection, and its knockdown decreases the number of sporozoites in mosquito salivary glands. These results together with the fact that glucose is a vital source of energy suggested that a glucose transporter is a candidate protective antigen for the control of mosquito infestations and Plasmodium infection. To address this hypothesis, herein we investigate the effect of mice vaccination with an immunogenic peptide from mosquito glucose transporter on Anopheles stephensi fitness and Plasmodium berghei infection. We showed that vaccination with a peptide of glucose transporter reduced mosquito survival by $5 \%$ when compared to controls. However, the reduction in Plasmodium infection was not significant in mosquitoes fed on vaccinated mice. The effect of the peptide vaccination on mosquito survival is important to reduce infestation by malaria vectors. These results support further research on developing glucose transporter-based vaccines to reduce mosquito fitness.
\end{abstract}

\section{Introduction}

Despite substantial progress in controlling malaria, the disease is still a public health problem in many countries, even with a notorious reduction on malaria cases and mortality rates of $41 \%$ and $62 \%$, respectively, between 2000 and 2015 [1]. The Global Technical Strategy for Malaria 2016-2030 defined as primary targets the reduction of the number of cases and deaths globally by at least $90 \%$ and the elimination and prevention of reestablishment of malaria from no less than 35 countries $[1,2]$.

Vaccines constitute the most efficacious intervention towards infectious diseases control. While some blood-stage vaccine candidates are presently under study, the Mosquirix or RTS,S/AS01 was already approved by the European
Medicine Agency and is recommended by the WHO for national immunization programs. This vaccine does not confer full protection against Plasmodium falciparum, the deadliest parasite causing human malaria, and, as such, it is often used in combination with other control measures, such as bed nets, indoor residual spraying, and antimalarial drugs, depending on disease prevalence and parasite resistance data from each country [3].

Transmission-blocking vaccines are a good example of an alternative/complementary control measure as these vaccines may disrupt the parasite life cycle in the Anopheles sp. mosquito. By reducing the number of infectious vectors and parasite reservoirs, the transmission of Plasmodium declines into the people community, leading to local group immunity $[4,5]$. The first malaria transmission-blocking vaccine were 
focused on surface antigens of parasite sexual stages but, recently, target antigens of the mosquito have also been investigated [6]. Due to the crucial role in parasite infection, Anopheles midgut or salivary gland-specific antigens have been used to interact with specific receptor-ligand peptides that are essential to block parasite invasion or maintenance [7-10].

Membrane transporter proteins are within the top five protein classes against which Food and Drug Administrationapproved drugs are developed [11]. These proteins encompass diverse gene families, namely, major facilitator superfamily transporters that allow shuttling of nutrient and metabolites, which can provide a regulated electrochemical gradient essential for mosquito survival [12]. As occurs with facilitated transporters and transmembrane integral proteins, glucose transporters are likely to be glycosylated [13] and this may modulate parasite recognition by the lobes of the mosquito salivary glands $[14,15]$, leading to parasite adhesion and/or invasion. Furthermore, these proteins allow passive passage of glucose across the cell membrane enabling the required metabolism of Plasmodium spp. for maintenance during sporogonic cycle in the vector [16].

The AGAP007752 gene, which encodes a glucose transporter (GT), is an example whose properties have been previously evaluated [17]. The gene coding for this GT had the highest expression in Plasmodium-infected salivary glands of A. coluzzii s.s. (A. gambiae molecular M form) in both RNAseq and relative-qPCR assays in comparison with noninfected groups [17], which suggests that the parasite is probably modulating AGAP007752 expression to overcome invasion and facilitate its maintenance in the target-organs $[14,16$, 18, 19]. Moreover, Pinheiro-Silva et al. (2015) showed that a significant reduction of AGAP007752 mRNA levels using interference RNA results in a reduction of the number of sporozoites by $44 \%$ in the salivary glands at 18 days after infection [17]. These findings emphasized the importance of AGAP007752 overexpression during Plasmodium infection and its potential as a candidate protective antigen for the development of a vaccine. Considering that malaria is a vector-borne disease a dual-effect vaccine is an attractive solution by targeting both mosquito and pathogen. In this way, research should focus not only on the effect of vaccination on transmission but also on the impact in mosquito biological processes such as oviposition and survival. Herein, we demonstrate that vaccination with an immunogenic and conserved peptide of the GT protein (GTp) reduced malaria vector survival suggesting its potential to be part of a multivalent vaccine for malaria control by reducing Plasmodium transmission.

\section{Materials and Methods}

2.1. Ethical Statement. All institutional (Instituto de Higiene e Medicina Tropical (IHMT) Ethical Committee and Divisão Geral de Alimentação e Veterinária (DAGV), $\operatorname{Art}^{\circ}$ 8, Portaria number 1005/92 of 23rd of October), national (DecretoLei number 129/92), and European (Directive 86/609/EEC) guidelines for the care and use of laboratory animals were followed. Animal procedures were carried out under approval number 023357 of DAGV.

2.2. Sequence Analysis. The amino acid sequence of AGAP007752 protein was used to search for the orthologues using Blastp (https://www.vectorbase.org/blast). A. darlingi, $A$. sinensis, and $A$. stephensi amino acid sequences were considered as orthologous and its sequences were obtained from the VectorBase database (https://www.vectorbase.org/ blast). All sequences were aligned with MAFFT (v7) [22] and all positions containing gaps were excluded with GUIDANCE2 [23]. Molecular Evolutionary Genetics Analysis (MEGA, version 6) software was used to obtain the best model to build the phylogenetic tree [21]. The Maximum Likelihood tree was constructed based on a bootstrapping method with 1000 replicates, LG model, and a proportion of gamma distributed sites [20]. The tree generated was visualized and edited using FigTree v1.4.3 [24].

\subsection{Prediction of Antigenic Determinants and Peptide Syn-} thesis. The linear epitope of AGAP007752 sequence was determined with near $75 \%$ accuracy based on the prediction of antigenic determinants using the method developed by Kolaskar and Tongaonkar [25] available at http://imed.med .ucm.es/Tools/antigenic.pl. A putative antigenic GTp was selected to be synthetically produced by ProteoGenix SAS Company (Schiltigheim, France) for the immunoassays and vaccine formulation.

2.4. Consensus Sequence Peptides. To check for similarity between the antigenic epitope of $A$. gambiae GT protein, previously selected, and the orthologue A. stephensi protein (ASTE006385), the ClustalW program (http://europepmc .org/abstract/MED/21988835) at the EBI website (http://www .ebi.ac.uk/Tools/msa/clustalo/) was used to align the amino acid sequences.

2.5. Vaccine Formulations. Lyophilized antigen with a positive net charge $(\mathrm{pH}=8.92)$ was reconstituted in $750 \mu \mathrm{L}$ of phosphate-buffered saline (PBS) and $50 \mu \mathrm{L}$ of acetic acid $0.6 \%$ $(\mathrm{v} / \mathrm{v})$ at a concentration of approximately $3.5 \mu \mathrm{g} / \mu \mathrm{l}$ peptide. The peptide-based vaccine composition was mixed 1:1 with Montanide ISA 50 V2 adjuvant (Seppic, France), at a final peptide concentration of $25 \mu \mathrm{g} / \mu \mathrm{l}$. A control was prepared as above but with PBS replacing the peptide.

2.6. Mice Vaccination and Infection Challenge. For immunization, twelve five-week-old female $\mathrm{Balb} / \mathrm{c}$ mice were obtained from Charles River Laboratories and kept in the IHMT animal facilities under controlled conditions. Mice from control group $(N=6)$ were primed and boosted intraperitoneally five times with $0.1 \mathrm{ml}$ each containing adjuvant vaccine, every fortnight. A vaccinated group $(N=6)$ was immunized with the GTp-formulated vaccine. Four days after the last immunization, mice were infected with $10^{7} P$. berghei ANKA parasitized red blood cells by intraperitoneal inoculation or left uninfected as control. 
2.7. Antibody Titer Determination. Before each immunization and four days after the last immunization, mouse tail blood was collected to obtain sera for determination of antiGTp antibodies titers by ELISA.

A high binding 96-well ELISA plate (Costar ${ }^{\circledR}$, MA, USA) was coated with $0.1 \mu \mathrm{g}$ of GTp protein diluted in $100 \mu \mathrm{l}$ PBS and incubated overnight at $4^{\circ} \mathrm{C}$. The plate was then washed five times with Tris buffered saline ( $25 \mathrm{mM}$ Tris $\mathrm{HCl}, 150 \mathrm{mM}$ $\mathrm{NaCl}$, and $2 \mathrm{mM} \mathrm{KCl}$ ) containing $0.05 \%(\mathrm{v} / \mathrm{v})$ Tween 20 (TBST), blocked with $300 \mu \mathrm{l}$ of $5 \%$ (w/v) milk (Bio-Rad, Hercules, CA, USA) at room temperature (RT) for 90 minutes and washed five times with TBST. For one hour at $37^{\circ} \mathrm{C}$, serum samples were incubated, and, after washing the plate, the secondary anti-mouse AP-conjugated immunoglobulins (Sigma-Aldrich, St. Louis, Missouri, USA) diluted 1:5,000 in TBST were added to each well for an incubation of one hour at $37^{\circ} \mathrm{C}$. Washed plates were incubated with $1 \mathrm{mg} / \mathrm{ml}$ of $\mathrm{p}-$ nitrophenyl phosphate in substrate buffer $(100 \mathrm{mM}$ glycine, $1 \mathrm{mM} \mathrm{MgCl} 2,1 \mathrm{mM} \mathrm{ZnCl}$, and $\mathrm{pH} 10.4)$ at $\mathrm{RT}$ in the dark. Plates were then read at a wavelength of $405 \mathrm{~nm}$ in an ELISA plate reader (Triad Series Multimode Detector, Dynex Technologies, Chantilly, VA, USA) with Concert-Triad Series software (version 2.1.0.17) at a wavelength $405 \mathrm{~nm}$. Negative and positive controls were included in the plates. Antibody titers were determined at a 1:500 serum dilution and were compared between GTp-immunized and control mice by Mann-Whitney test $(P<0.05)$ (SPSS v24.0) [26].

2.8. Mosquito Rearing. A. stephensi mosquitoes (SDA-500 strain) were obtained from the IHMT insectary, reared at $20^{\circ} \mathrm{C}$ in $70 \%$ humidity, under a $12 \mathrm{~h}$ light/dark photoperiod, and fed ad libitum on a $10 \%$ glucose solution. For experiments, only mosquitoes aged between 3 and 5 days were used.

2.9. Anopheles stephensi Infection with Plasmodium berghei. Mice blood samples were collected from mouse tail to determine parasitemia, using light microscopy after staining with Hemacolor ${ }^{\circledR}$ kit (EMD Millipore, Germany). When the parasitaemia reached $10-20 \%$ and $4-6$ exflagellations/field were observed, mice were anesthetized and used to feed and infect female mosquitoes ( $N=300 /$ mice $)$. Unfed female mosquitoes were excluded, while fully engorged mosquitoes were maintained at $19-21^{\circ} \mathrm{C}$ and $80 \%$ humidity for $P$. berghei development.

After blood meal, mosquitoes were kept in the cage with egg cups for oviposition evaluation. To count the number of eggs within groups, images from egg cups were taken and analyzed using ImageJ 1.50i [27]. Mosquito survival for 18 days after feeding was also evaluated by counting the number of survived mosquitoes between groups. Results from mosquitoes fed on GTp-immunized and control mice were compared by Mann-Whitney test $(P<0.05)$ (SPSS v24.0) [26].

2.10. Determination of Plasmodium Infection. To check for rates in parasite infection, at day 18 after blood-meal, cold anaesthetized female mosquitoes were used and salivary glands dissected to perform RNA extraction. RNA was immediately extracted using GRS FullSample Purification Kit (GRiSP, Porto, Portugal), quantified using a ND-1000 Spectrophotometer (NanoDrop ND1000, Thermo Fisher Scientific, Waltham, MA), and stored at $-80^{\circ} \mathrm{C}$. Using three biological replicates of each condition (immunized or nonimmunized), total RNA (100 ng/ $\mu \mathrm{L})$ was used to synthesize cDNA using the iScript ${ }^{\mathrm{TM}}$ cDNA Synthesis Kit (Bio$\mathrm{Rad}, \mathrm{CA}, \mathrm{USA})$. qPCR reactions of $10 \mu \mathrm{l}$ were performed in triplicate using $\mathrm{IQ}^{\mathrm{TM}} \mathrm{SYBR}^{\circledR}$ Green Supermix kit (BioRad, CA, USA) in a CFX96 Touch Real-Time PCR (BioRad, CA, USA) thermocycler. The following conditions were used: an initial cycle of denaturation at $95^{\circ} \mathrm{C}$ for $10 \mathrm{~min}$, followed by 45 cycles of $95^{\circ} \mathrm{C}$ for 15 seconds and $55^{\circ} \mathrm{C}$ for 45 seconds to amplify the target Plasmodium 18s rRNA gene. Fluorescence readings were taken at $62^{\circ} \mathrm{C}$ after each cycle and a melting curve $\left(60-95^{\circ} \mathrm{C}\right)$ was performed. To determine the reaction efficiency, standard curves were constructed with 5fold serial dilutions of cDNA synthetized from total RNA from a pool of samples. Relative expression levels mosquito samples were normalized using the ribosomal protein S7 $\left(60^{\circ} \mathrm{C}\right)$ as reference gene and gene expression was analyzed using the CFX Manager ${ }^{\mathrm{TM}}$ Software version 3.1 (Bio-Rad, CA, USA).

2.11. Immunofluorescence Assay. To evaluate the serum ability to detect the native antigen on A. stephensi salivary glands, immunofluorescence assays (IFA) were performed. Salivary glands were dissected and stored at $4^{\circ} \mathrm{C}$ in $4 \%$ (w/v) paraformaldehyde solution (Sigma-Aldrich, St. Louis, Missouri, USA) diluted in PBS. For immunolocalization, stored tissues were placed in glass slides (BioMérieux, France) and washed three times for 15 minutes with PBS. Salivary glands were subsequently permeabilized with $0.2 \%(\mathrm{v} / \mathrm{v})$ Triton X-100 (USB, Cleveland, OH, USA) in PBS for 30 minutes at RT and washed with PBS (three times for 15 minutes) and a blocking solution of 3\% (w/v) bovine serum albumin (BSA) (Sigma-Aldrich, St. Louis, Missouri, USA) in PBS was added for $30 \mathrm{~min}$ at RT. After a washing step, PBS, primary antibody from nonimmunized mice and primary antibody from immunized mice, diluted $(1: 100)$ in blocking solution, was applied to respective wells and the slides were incubated overnight at $4^{\circ} \mathrm{C}$ in the dark. The secondary antibody, Alexa Fluor ${ }^{\circledR} 647$ (magenta) from rabbit anti-mouse IgG $(\mathrm{H}+\mathrm{L})$ (Molecular Probes, Thermo Fisher Scientific, Waltham, Massachusetts, United States), diluted $(1: 100)$ in blocking solution was incubated for one hour at RT. After three PBS washes, a drop of ProLong ${ }^{\circledR}$ Gold Antifade Reagent with $4^{\prime}, 6^{\prime}$-diamidino-2-phenylindole (DAPI) (Invitrogen, Carlsbad, CA, USA) was placed over the tissues and then slides were sealed with a coverslip. After mounting, slides were placed in a humid dark box until microscopy analysis to prevent drying and fluorescence fading.

Sections of salivary glands were examined and photographed under $400 \mathrm{x}$ original magnification of a Nikon eclipse 80i fluorescence microscope with Nikon DS-Ril camera (Nikon Europe, Amsterdam, Netherlands) and appropriate filters (GFP/DAPI/CY5). Images were saved as high 


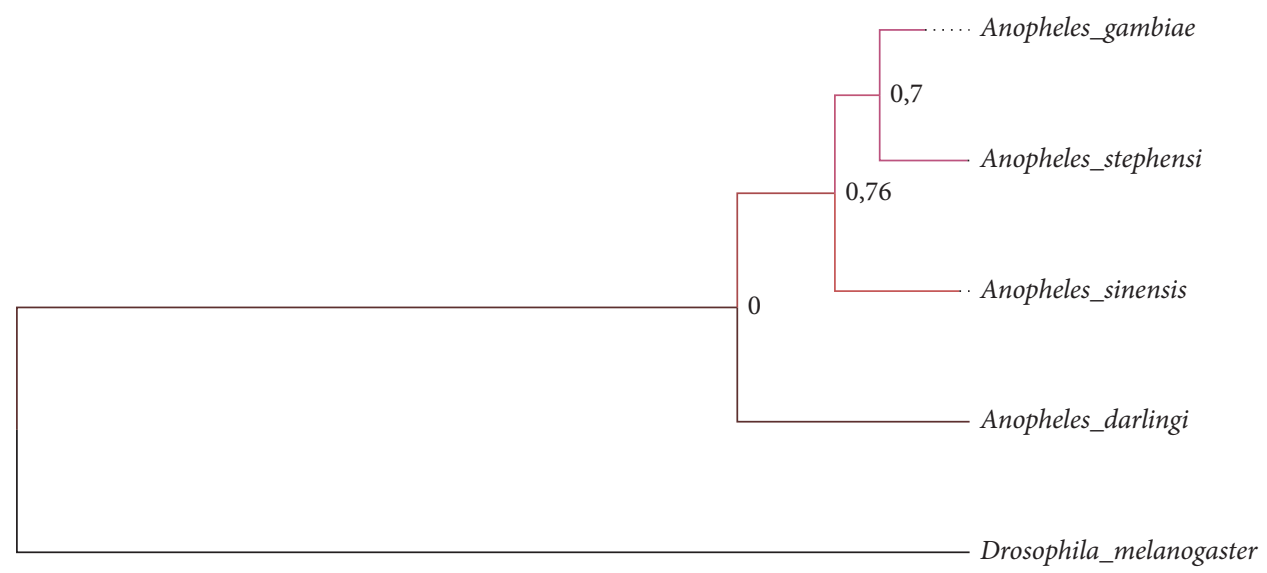

0.1

Figure 1: Molecular Phylogenetic Analysis by Maximum Likelihood Method. Using the Maximum Likelihood method based on the Le_Gascuel_2008 model [20], the tree with the highest log likelihood (-3359.935) is shown. A discrete Gamma distribution was used to model evolutionary rate differences among sites $(5$ categories $(+\mathrm{G}$, parameter $=1.65)$ ). The tree is drawn to scale, with branch lengths measured in the number of substitutions per site. The analysis involved 5 amino acid sequences with respective VectorBase accession number: AGAP007752: Anopheles gambiae, ASTE006385: A. stephensi, ASIS016443: A. sinensis, ADAC010569: A. darlingi, and CG15406: Drosophila melanogaster (outgroup). All positions containing gaps and missing data were eliminated and bootstrap values for internal branches are shown. Evolutionary analyses were conducted in MEGA6 [21].

quality TIFF files using NIS-ELEMENTS BR 3.2 (Nikon Europe, Amsterdam, Netherlands) software, and the fluorescence intensity was compared between sections by ImageJ 1.50i [27]. Experiments were conducted in parallel and the image acquisition parameters were the same.

\section{Results and Discussion}

3.1. Glucose Transporter in Anophelines. When searching for potential targets to develop a mosquito stage vaccine, receptors or transmembrane transporters are considered important molecules to interrupt malaria transmission by targeting parasite adhesion and/or invasion and/or maintenance in the mosquito tissues $[28,29]$.

To determine the potential of AGAP007752 as a new malaria control approach, we hypothesized that such gene modulation could occur in other vectors of malaria, such as A. stephensi and may interfere in Plasmodium infection and vector sustainability.

Sequence alignment analysis demonstrates that the $A$. gambiae GT is closely related to the A. stephensi protein (ASTE006385) sharing 84\% identical residues. Moreover, the A. gambiae amino acid sequence shares a high similarity with the $A$. sinensis (82\%) and A. darlingi (70\%). The evolutionary history of the AGAP007752 protein was inferred (Figure 1). These results highlight the high degree of conservation of GT between anophelines and the potential to use this target to block malaria transmission in different malaria vector species.

Both AGAP007752 and ASTE006385 proteins belong to the major facilitator superfamily responsible for the binding and transport of sugar molecules (InterPro: IPR005829 and IPR005828) [30]. And, as mentioned in previous studies, GT is a highly conserved transporter protein with 12 transmembrane domains and a unique N-linked oligosaccharide side-chain present in a large extracellular loop [17]. From all the predicted antigenic peptides of this protein, the longest sequence with high polarity (hydrophilic) and $80 \%$ identity with the $A$. stephensi protein (ASTE006385) and located between amino acids 190 and 260 (PDTPQTCLKKGRTAEAERSFMFYRGIRTQAEKTSALRQEFDNMEKFIEHNSGQNSRVTLADFKSREAKLGI) was selected for synthesis. The synthetic peptide with a molecular weight of $8.17 \mathrm{KDa}$ and a purity of $94.5 \%$ was then used for immunization assays using $A$. stephensi mosquitoes.

3.2. Localization of the GT Conserved Peptide in A. stephensi during $P$. berghei Infection. An immunofluorescence assay was performed to characterize the presence and localization of GT in A. stephensi salivary glands during Plasmodium infection (Figure 2). As negative control, nucleated and infected SGs-cells were incubated with PBS and led to no reaction in Cy5 channel. When preimmune serum was added, a diffuse fluorescence was observed in the Z-stack images related to a nonspecific interaction between several proteins and the polyclonal serum. As observed in Figure 2, the fluorescence labeling is higher and specific on Cy5 filter with anti-GTp serum. These results confirm the specific interaction of antibodies with GTp in infected salivary glands of $A$. stephensi, distributed in the membrane heterogeneously.

These results corroborated the presence of GT in $A$. stephensi SGs, which is in accordance with previous reports of GT proteins as a key for parasite recognition in the salivary glands $[14,15]$, providing a regulated electrochemical 


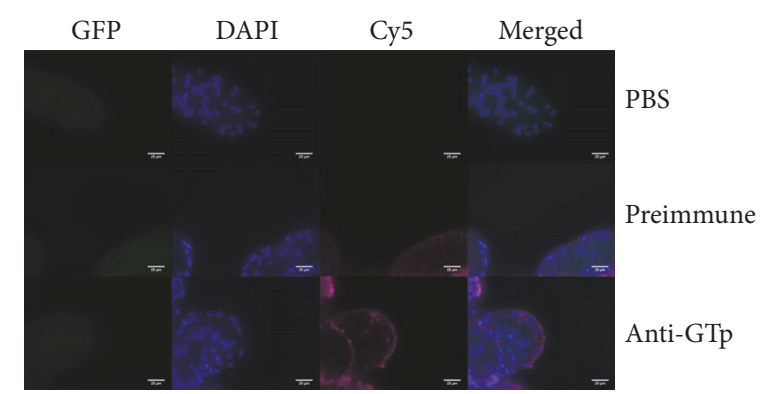

FIgure 2: Fluorescence Microscopy of A. stephensi-Infected Salivary Glands Triple-Stained. Salivary glands were incubated with PBS, preimmune and anti-GTp immune serum. Green fluorescence corresponds to GFP-expressing $P$. berghei, blue to the nuclei stained with DAPI and magenta for Cy5-Alexa Fluor 647 to localize specific anti-GTp antibodies. The fluorescence intensity of SG Z-stack and merged images was compared and then analyzed in the same conditions. A specific and localized recognition was evident when SGs were incubated with the anti-GTp serum. Z-stack maximum intensity projections and a merge image of the three channels are given. Green: GFP-expressing P. berghei. Blue: DAPI-counterstaining of the nuclei. Magenta: Cy5-Alexa Fluor 647. Bar: $25 \mu \mathrm{m}$.

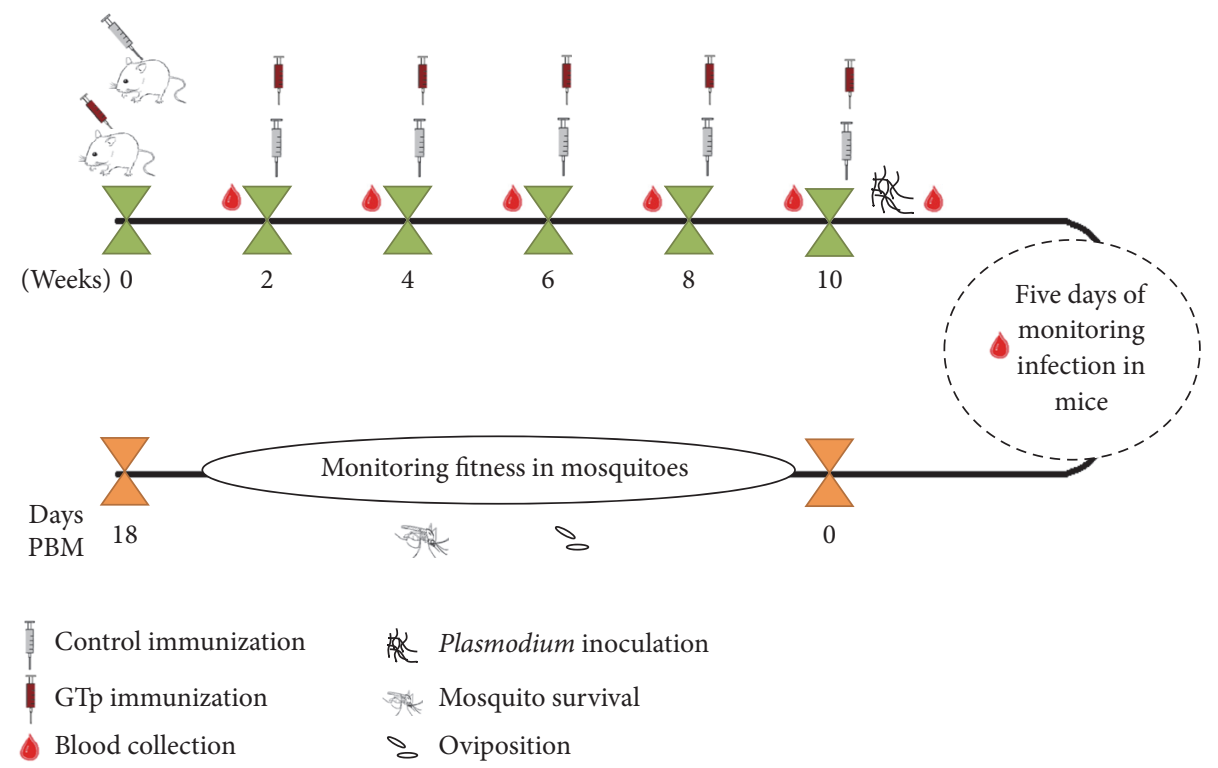

FIGURE 3: Schematic Overview of Vaccination Trial. From twelve five-week-old female Balb/c mice, six were primed with PBS and the others with GTp-formulated vaccine. Every fortnight, mice were boosted five times intraperitoneally. Four days after the last immunization, mice were infected with $P$. berghei parasitized red blood cells by intraperitoneal inoculation or left uninfected as control. Blood samples were collected to determinate antibody titers during immunization and after Plasmodium infection. For five days the infection was monitored and when the parasitaemia reached $10-20 \%$ and $4-6$ exflagellations/field were observed, mice were anesthetized and used to feed and infect female mosquitoes $(N=300 /$ mice). Mosquito survival and oviposition were assessed until the 18th day after blood meal (PBM).

gradient and nutrients to cell and parasite maintenance [12].

3.3. Impact of GTp Immunization on Mosquito Infestations and Parasite Infection. Vaccination trials were conducted to elucidate the impact of GTp on mosquito oviposition and mortality and $P$. berghei parasite infection of salivary glands (Figure 3).

During subsequent GTp immunizations, the antibody titers started to increase, even after pathogen inoculation in mice (Figure 4), revealing a successful immune response to GTp administration and no consequence in the parasite erythrocytic cycle, as expected for an Anopheles-specific antigen.

Immune-stimulated mice with or without Plasmodium infection were used for female A. stephensi mosquitoes blood meal containing Plasmodium parasites and antibodies against salivary GTp, and, then, the oviposition (Figure 5(a)), mosquito survival (Figure 5(b)), and parasite infection (Figure 5(c)) were assessed.

As expected, as a carbohydrate and precursor in the synthesis of glycogen, trehalose [31], and lipids [32], glucose is 


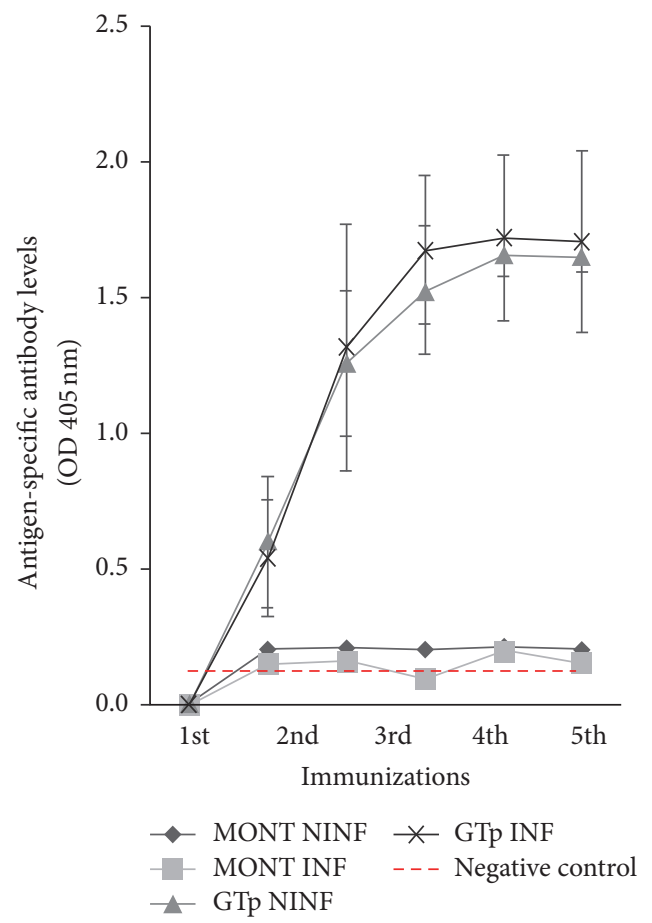

Figure 4: Mice Immunization with GTp after Challenge or Not with P. berghei. Comparison between GTp-immunized and control mice with and without Plasmodium infection. Antibody titers were determined by ELISA assays and Plasmodium inoculation is represented with a red arrow. Negative control is represented in a red dashed line. Statistical analyses were performed using Mann-Whitney test $(P<0.05)$. MONT NINF: control mice without Plasmodium infection; MONT INF: control mice with Plasmodium infection; GTp NINF: GTp-immunized mice without Plasmodium infection; GTp INF: GTp-immunized mice with Plasmodium infection.

essential for insect development, metabolism, and oogenesis [33]. Therefore, disequilibrium in the levels of this energetic component could alter survival, mating, host seeking, and oviposition behaviors [34]. Besides, an impact on this pathway could slightly decrease Plasmodium infection and consequently improve the mosquito fitness.

Such imbalance probably happened during vaccination trials, where anti-GTp antibodies interacted with the target protein in A. stephensi salivary glands leading to a significant increase of about $29 \%$ in the number of the eggs $(P=0.05)$ (Figure 5(a)) and an average reduction of $4.67 \%$ in mosquito survival during all replicas with significant impact between the 5th and the 18th day (Log Rank/Mantel-Cox: $P=0.085$; Breslow/Generalized Wilcoxon: $P<0.0001$; Tarone-Ware: $P=0.003$ ) (Figure 5(b)). Further studies could evaluate eggs viability to focus on the effect of vaccination in the quantity of glucose-metabolites inside the egg and subsequently egg resistance desiccation effect [35].

Regarding the interference of GTp in Plasmodiuminfected SGs, our results point to a no statistical reduction in $P$. berghei mRNA levels in the A. stephensi tissue (Figure 5(c)). We believe that using higher amount of antibodies anti-GTp acquisition by mosquitoes or even immunizing mice with the full length protein would significantly increase the reduction of the infection. Moreover, posttranslational modifications like glycosylation, often occurring in GTs, cannot be ruled out as potential efficiency promoter on the design of vaccines by increasing protein immunogenicity.

\section{Conclusions}

To break the complex interplay during malaria parasite transmission, a mosquito stage vaccine that works in tandem with other interventions would be a critical tool for malaria elimination/eradication. GTp is a conserved and immunogenic peptide that reduces $A$. stephensi survival, therefore suggesting its potential for the control of different malaria vectors. Further researchs on developing GT-based vaccines need to be conducted to reduce mosquito survival and parasite infection/multiplication.

\section{Conflicts of Interest}

The authors declare that there are no conflicts of interest regarding the publication of this article.

\section{Authors' Contributions}

S. Antunes, J. Couto, J. de la Fuente, and A. Domingos designed the study; J. Couto performed the mosquito experiments and molecular work. All authors performed data analysis and wrote the manuscript. All authors edited and approved the final manuscript.

\section{Acknowledgments}

The authors would like to thank Dr. Pedro Cravo and Dr. Alejandro Cabezas-Cruz for their advice. This work 


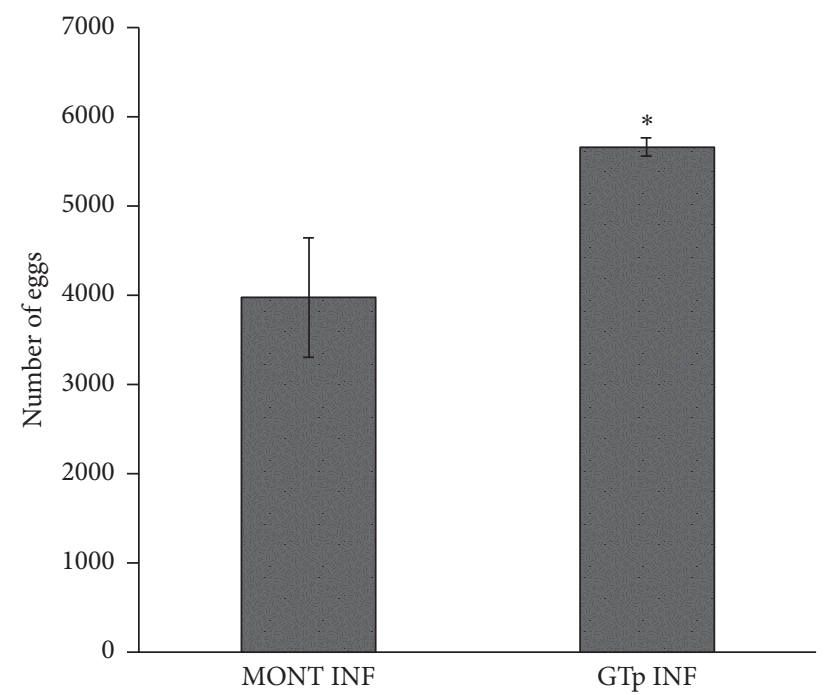

(a)

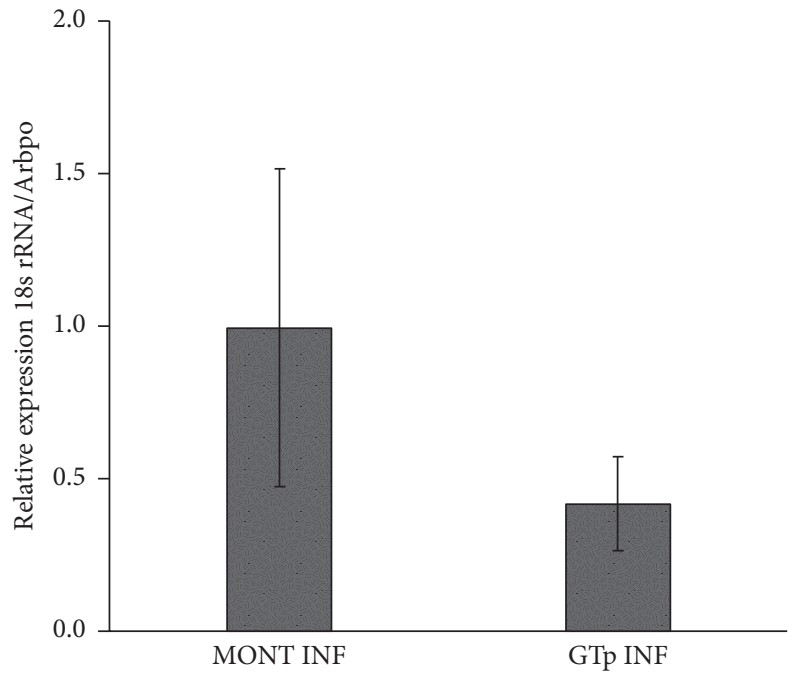

(c)

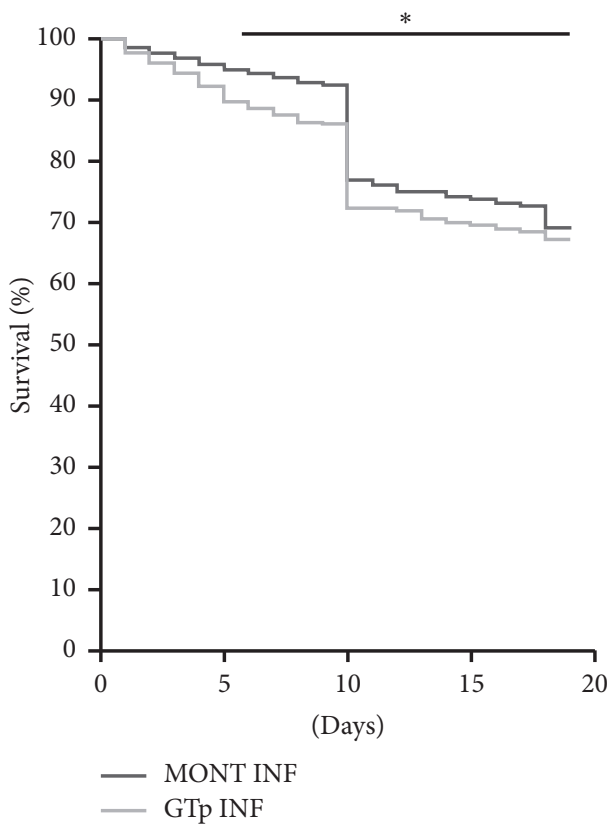

(b)

Figure 5: The Effect of Anti-GTp Antibodies on A. stephensi Mosquitos and Malaria Parasites. (a) Oviposition. Total number of eggs laid after each experiment $(N=3)$ were counted and compared between GTp-immunized and nonimmunized groups. (b) Mosquito survival after blood meal with anti-GTp immune serum and preimmune serum. (c). Plasmodium mRNA levels at 18th day after blood meal in SGs tissues. Statistical analyses of (a) and (b) results were performed using Mann-Whitney test and for (c) using CFX Manager Software $\left({ }^{*} P<0.05\right)$. MONT INF: control mice with Plasmodium infection; GTp INF: GTp-immunized mice with Plasmodium infection.

was partially supported by Fundação para a Ciência e a Tecnologia-Global Health and Tropical Medicine (FCTGHTM) [UID/Multi/04413/2013].

\section{References}

[1] WHO, "World Malaria Report 2016," 2016, http://apps.who.int/ iris/bitstream/10665/252038/1/9789241511711-eng.pdf?ua=1.

[2] WHO, "World Malaria Report 2015," 2015, http://apps.who.int/ iris/bitstream/10665/200018/1/9789241565158_eng.pdf?ua=1.

[3] S. T. Agnandji, B. Lell, J. F. Fernandes et al., "Efficacy and safety of the RTS,S/AS01 malaria vaccine during 18 months after vaccination: a phase 3 randomized, controlled trial in children and young infants at 11 African sites," PLoS Medicine, vol. 11, no. 7, Article ID e1001685, 2014.

[4] A. Saul, "Efficacy model for mosquito stage transmission blocking vaccines for malaria," Parasitology, vol. 135, no. 13, pp. 1497-1506, 2008.

[5] S. C. Atkinson, J. S. Armistead, D. K. Mathias et al., "The Anopheles-midgut APN1 structure reveals a new malaria transmissionblocking vaccine epitope," Nature Structural and Molecular Biology, vol. 22, no. 7, pp. 532-539, 2015.

[6] R. R. Dinglasan and M. Jacobs-Lorena, "Flipping the paradigm on malaria transmission-blocking vaccines," Trends in Parasitology, vol. 24, no. 8, pp. 364-370, 2008. 
[7] S. Korochkina, C. Barreau, G. Pradel et al., "A mosquito-specific protein family includes candidate receptors for malaria sporozoite invasion of salivary glands," Cellular Microbiology, vol. 8, no. 1, pp. 163-175, 2006.

[8] J. M. Myung, P. Marshall, and P. Sinnis, "The Plasmodium circumsporozoite protein is involved in mosquito salivary gland invasion by sporozoites," Molecular and Biochemical Parasitology, vol. 133, no. 1, pp. 53-59, 2004.

[9] R. Rosenberg, "Inability of Plasmodium Knowlesi sporozoites to invade Anopheles freeborni salivary glands," The American Journal of Tropical Medicine and Hygiene, vol. 34, no. 4, pp. 687691, 1985.

[10] J. S. Armistead, I. Morlais, D. K. Mathias et al., "Antibodies to a single, conserved epitope in anopheles APN1 inhibit universal transmission of Plasmodium falciparum and Plasmodium vivax malaria," Infection and Immunity, vol. 82, no. 2, pp. 818-829, 2014.

[11] J. P. Overington, B. Al-Lazikani, and A. L. Hopkins, "How many drug targets are there?” Nature Reviews Drug Discovery, vol. 5, no. 12, pp. 993-996, 2006.

[12] S. Kenthirapalan, A. P. Waters, K. Matuschewski, and T. W. A. Kooij, "Functional profiles of orphan membrane transporters in the life cycle of the malaria parasite," Nature Communications, vol. 7, Article ID 10519, 2016.

[13] G. Overend, Drosophila as a Model for the Anopheles Malpighian Tubule, University of Glasgow, 2010.

[14] R. R. Dinglasan, J. G. Valenzuela, and A. F. Azad, "Sugar epitopes as potential universal disease transmission blocking targets," Insect Biochemistry and Molecular Biology, vol. 35, no. 1, pp. 1-10, 2005.

[15] G. E. Zollner, N. Ponsa, G. W. Garman et al., "Population dynamics of sporogony for Plasmodium vivax parasites from western Thailand developing within three species of colonized Anopheles mosquitoes," Malaria Journal, vol. 5, article 68, 2006.

[16] K. Liu, Y. Dong, Y. Huang, J. L. Rasgon, and P. Agre, "Impact of trehalose transporter knockdown on Anopheles gambiae stress adaptation and susceptibility to Plasmodium falciparum infection," Proceedings of the National Academy of Sciences of the United States of America, vol. 110, no. 43, pp. 17504-17509, 2013.

[17] R. Pinheiro-Silva, L. Borges, L. P. Coelho et al., "Gene expression changes in the salivary glands of Anopheles coluzzii elicited by Plasmodium berghei infection," Parasites and Vectors, vol. 8, no. 1, article 485, 2015.

[18] H. Zieler and J. Nawrocki, "Plasmodium gallinaceum ookinetes adhere specifically to the midgut epithelium of Aedesaegypti by interaction with a carbohydrate ligand," Journal of Experimental Biology, vol. 202, pp. 485-495, 1999.

[19] H. R. Basseri, S. Doosti, K. Akbarzadeh, M. Nateghpour, M. M. A. Whitten, and H. Ladoni, "Competency of Anopheles stephensi mysorensis strain for Plasmodium vivax and the role of inhibitory carbohydrates to block its sporogonic cycle," Malaria Journal, vol. 7, article 131, 2008.

[20] S. Q. Le and O. Gascuel, "An improved general amino acid replacement matrix," Molecular Biology and Evolution, vol. 25, no. 7, pp. 1307-1320, 2008.

[21] K. Tamura, G. Stecher, D. Peterson, A. Filipski, and S. Kumar, "MEGA6: molecular evolutionary genetics analysis version 6.0," Molecular Biology and Evolution, vol. 30, no. 12, pp. 2725-2729, 2013.

[22] K. Katoh and D. M. Standley, "A simple method to control over-alignment in the MAFFT multiple sequence alignment program," Bioinformatics, vol. 32, no. 13, pp. 1933-1942, 2016.
[23] I. Sela, H. Ashkenazy, K. Katoh, and T. Pupko, "GUIDANCE2: Accurate detection of unreliable alignment regions accounting for the uncertainty of multiple parameters," Nucleic Acids Research, vol. 43, no. 1, pp. W7-W14, 2015.

[24] A. Rambaut, FigTree, 2006, http://tree.bio.ed.ac.uk/software/ figtree/.

[25] A. S. Kolaskar and P. C. Tongaonkar, "A semi-empirical method for prediction of antigenic determinants on protein antigens," FEBS Letters, vol. 276, no. 1-2, pp. 172-174, 1990.

[26] IBM Corporation. IBM SPSS Statistics for Windows, 2016.

[27] C. A. Schneider, W. S. Rasband, and K. W. Eliceiri, "NIH Image to ImageJ: 25 years of image analysis," Nature Methods, vol. 9, no. 7, pp. 671-675, 2012.

[28] J. L. Ramirez, L. S. Garver, and G. Dimopoulos, "Challenges and approaches for mosquito targeted malaria control," Current Molecular Medicine, vol. 9, no. 2, pp. 116-130, 2009.

[29] V. VenkatRao, S. K. Kumar, P. Sridevi, V. Y. Muley, and R. Chaitanya, "Cloning, characterization and transmission blocking potential of midgut carboxypeptidase A in Anopheles stephensi," Acta Tropica, vol. 168, pp. 21-28, 2017.

[30] A. Mitchell, H.-Y. Chang, L. Daugherty et al., "The InterPro protein families database: the classification resource after 15 years," Nucleic Acids Research, vol. 43, no. 1, pp. D213-D221, 2015.

[31] P. D. Evans, G. A. Kerkut, and L. Gilbert, Comprehensive Insect Biochemistry, Physiology and Pharmacology, Pergamon Press, New York, NY, USA, 1st edition, 1985.

[32] E. Van Handel and P. T. M. Lum, "Sex as regulator of triglyceride metabolism in the mosquito," Science, vol. 134, no. 3494, pp. 1979-1980, 1961.

[33] J. Steele, The Role of Carbohydrate Metabolism in Physiological Function, Springer, Boston, Mass, USA, 1981.

[34] M. C. Hardstone, X. Huang, L. C. Harrington, and J. G. Scott, "Differences in development, glycogen, and lipid content associated with cytochrome $\mathrm{P} 450$-mediated permethrin resistance in Culex pipiens quinquefasciatus (Diptera: Culicidae)," Journal of Medical Entomology, vol. 47, no. 2, pp. 188-198, 2010.

[35] L. C. Farnesi, R. F. S. Menna-Barreto, A. J. Martins, D. Valle, and G. L. Rezende, "Physical features and chitin content of eggs from the mosquito vectors Aedes aegypti, Anopheles aquasalis and Culex quinquefasciatus: connection with distinct levels of resistance to desiccation," Journal of Insect Physiology, vol. 83, pp. 43-52, 2015. 

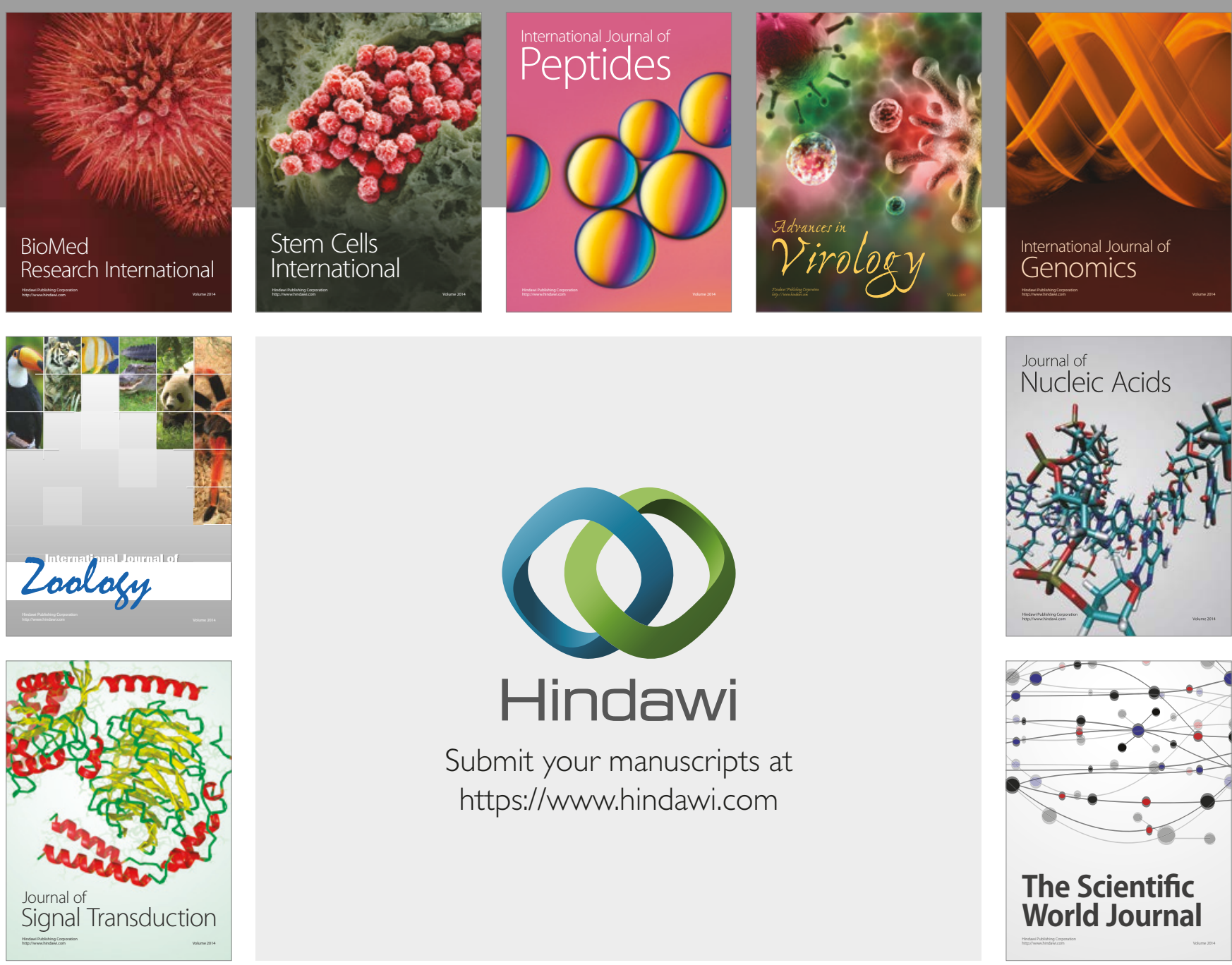

Submit your manuscripts at

https://www.hindawi.com
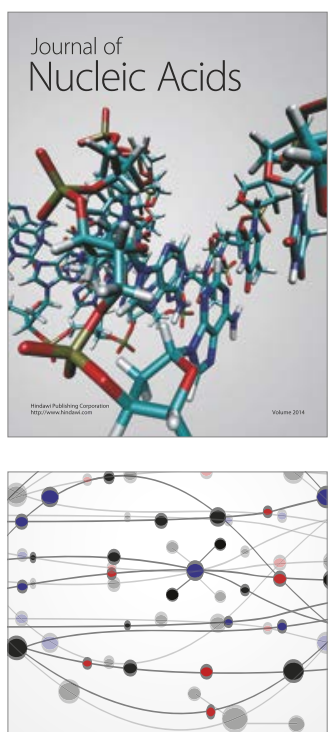

The Scientific World Journal

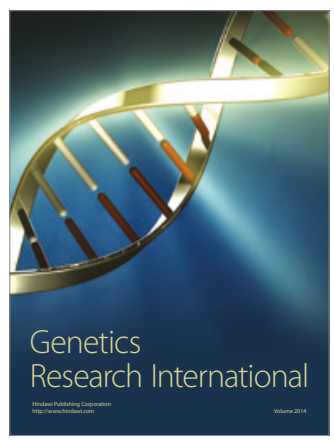

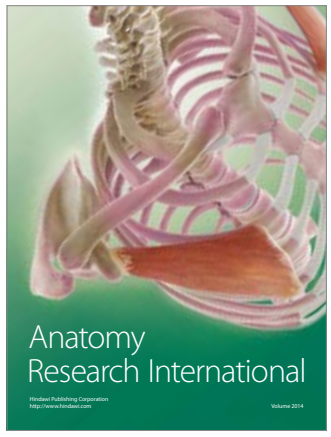

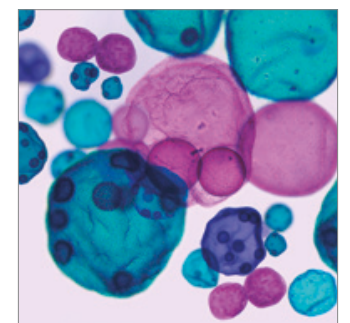

International Journal of Microbiology
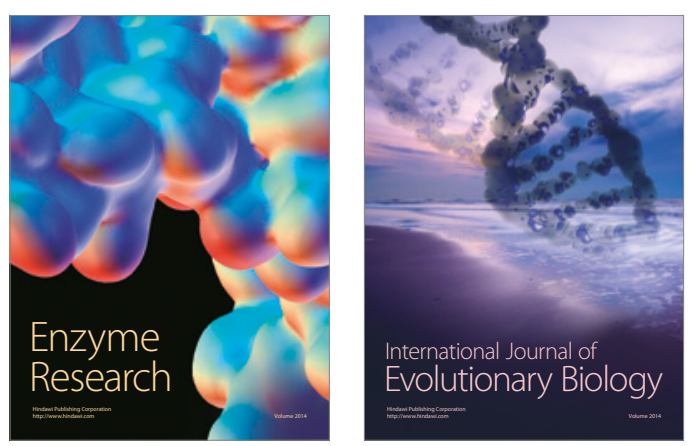
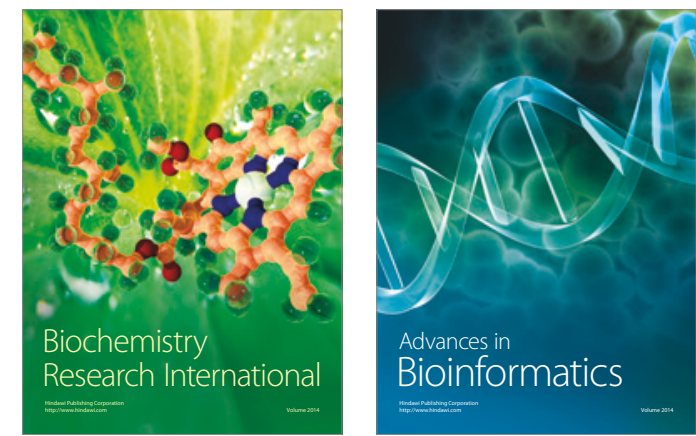

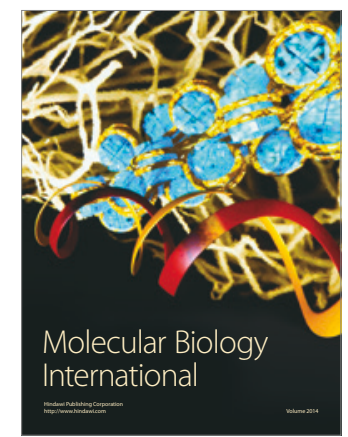

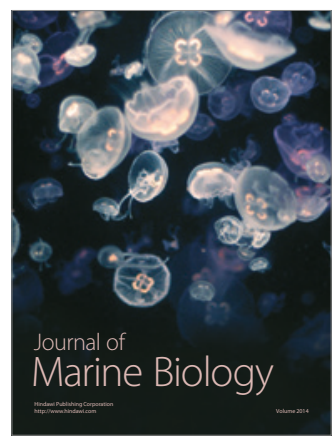

